\title{
Pancreatic Allograft Thrombosis: Suggestion for a CT Grading System and Management Algorithm
}

\author{
Hakeem $\mathrm{A}^{1}$, Chen $\mathrm{J}^{1}$, lype $\mathrm{S}^{1}$, Clatworthy $\mathrm{M}^{2}$, Watson $\mathrm{C}^{1}$, Godfrey $\mathrm{E}^{3}$, Upponi $\mathrm{S}^{3}$, Saeb-Parsy $\mathrm{K}^{1}$ \\ Department of Surgery ${ }^{1}$, Department of Medicine², Department of Radiology3; University of Cambridge and NIHR Cambridge \\ Biomedical Research Centre; Cambridge University Hospitals NHS Trust, Hills Road, Cambridge, CB2 OQQ
}

\section{Introduction:}

- Pancreatic allograft thrombosis remains the leading cause of nonimmunological graft failure.

- There is currently no consensus on the reporting and management of partial pancreatic allograft thrombosis.

- Herein we propose a new CT grading system of pancreatic allograft thrombosis for identification of patients at risk of allograft loss and outline a management algorithm.

\section{Methods:}

- Retrospective review of all consecutive pancreatic transplants performed at our centre between 2009 and 2014.

- Triple phase CT scans were retrospectively graded independently by two radiologists as;

Grade 0 - No allograft thrombosis
Grade I - Tiny distal or peripheral thrombosis
Grade 2 - Intermediate non-occlusive thrombosis
Grade 3 - Proximal and occlusive thrombosis

\section{Results:}

- 103 consecutive pancreatic transplants were performed, of which 24 (23.3\%) were diagnosed with allograft thrombosis.

- Three grafts were lost due to thrombosis [all portal vein thrombosis (two grade 3 and one grade 2);2.9\%].

- All 24 patients were started on therapeutic anticoagulation.

- Re-exploration rate for bleeding was $33.3 \%$ for thrombosis group and $15.2 \%$ for no thrombosis group.

- On multivariate analysis, pancreas after kidney transplant (OR I.09, Cl 0.0I-0.97, $p=0.047$ ), acute rejection (OR I.25, $\mathrm{Cl}$ 0.07-0.90, $p=0.034$ ) and $\mathrm{CT}$ finding of pancreatitis (perigraft fat stranding) (OR I.23, Cl 0.08-0.72, $\mathrm{p}=0.0 \mathrm{II}$ ) were risk factors of allograft thrombosis.

- Retrospective review of CT images revealed more grade I and 2 thromboses than were initially reported.

Four grade 2 and one grade 3 arterial thrombosis were
anticoagulated without exploration and all have functioning grafts.
Two grade 2 and two grade 3 venous thrombosis were
re-explored, of which two patients needed pancreatectomy.
One grade 2 and two grade 3 venous thrombosis were
anticoagulated and all patients have functioning grafts.

There was no difference in graft or patient
survival, length of stay or morbidity between the
patients with grade $I$ and 2 thrombosis who were
anticoagulated $(n=I I)$ and with those who were
not anticoagulated $(n=26)$

\section{Discussion:}

- Based on our proposed grading system, our data suggest that grade I and 2 arterial thrombosis and grade I venous thrombosis can be managed safely without formal anticoagulation, thereby lowering the risk of immediate post-operative bleeding complications and associated morbidity.

- The proposed grading system will assist clinicians in decision making and can be used in future studies to provide standardised scoring to further interrogate the impact of thrombosis on outcome.

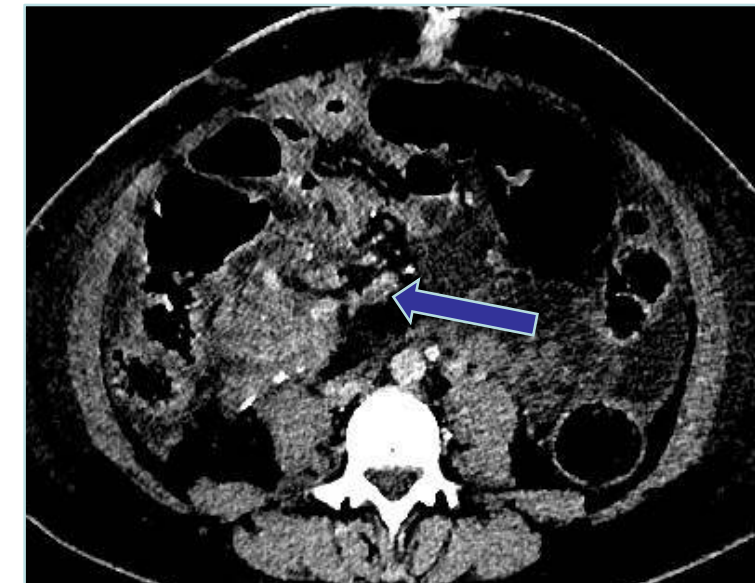

Grade I venous - small volume of thrombus in the distal SMV

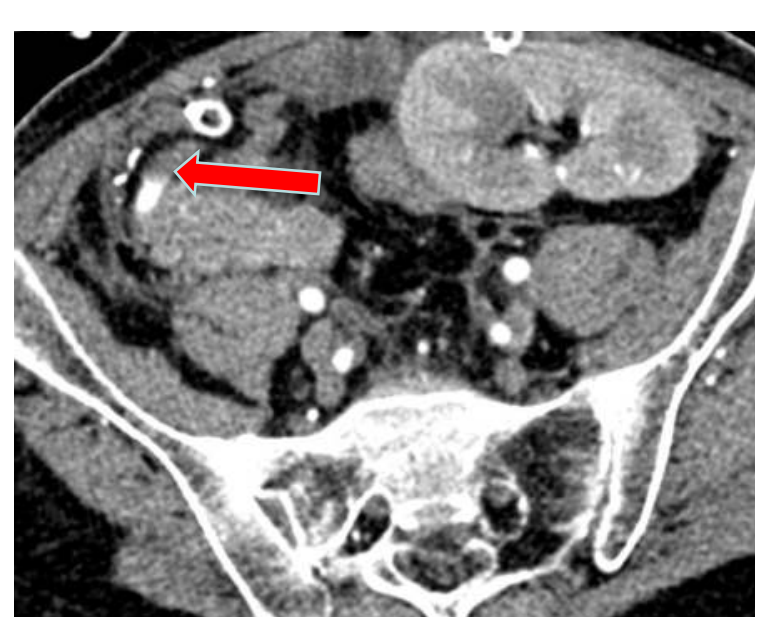

Grade I arterial - minimal thrombus in the distal SA

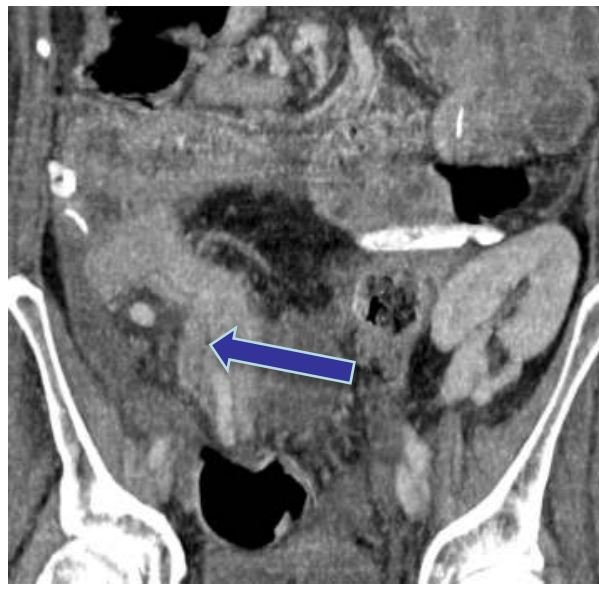

Grade 2 venous -

thrombus in the distal SV

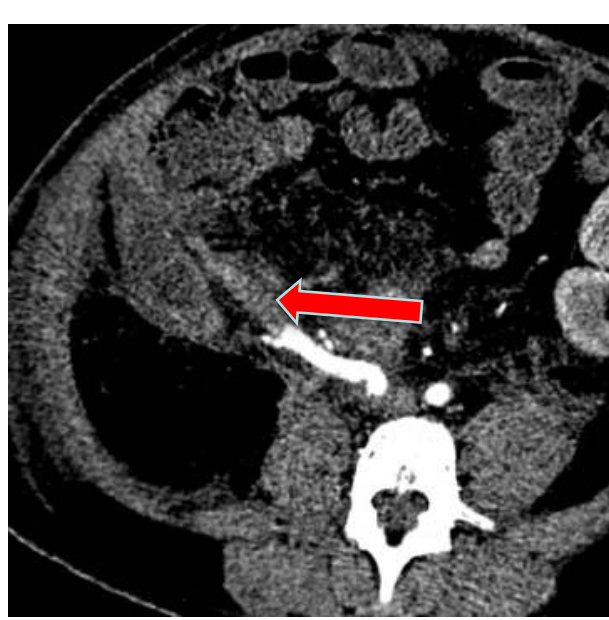

Grade 2 arterial - thrombus extending into the mid SMA
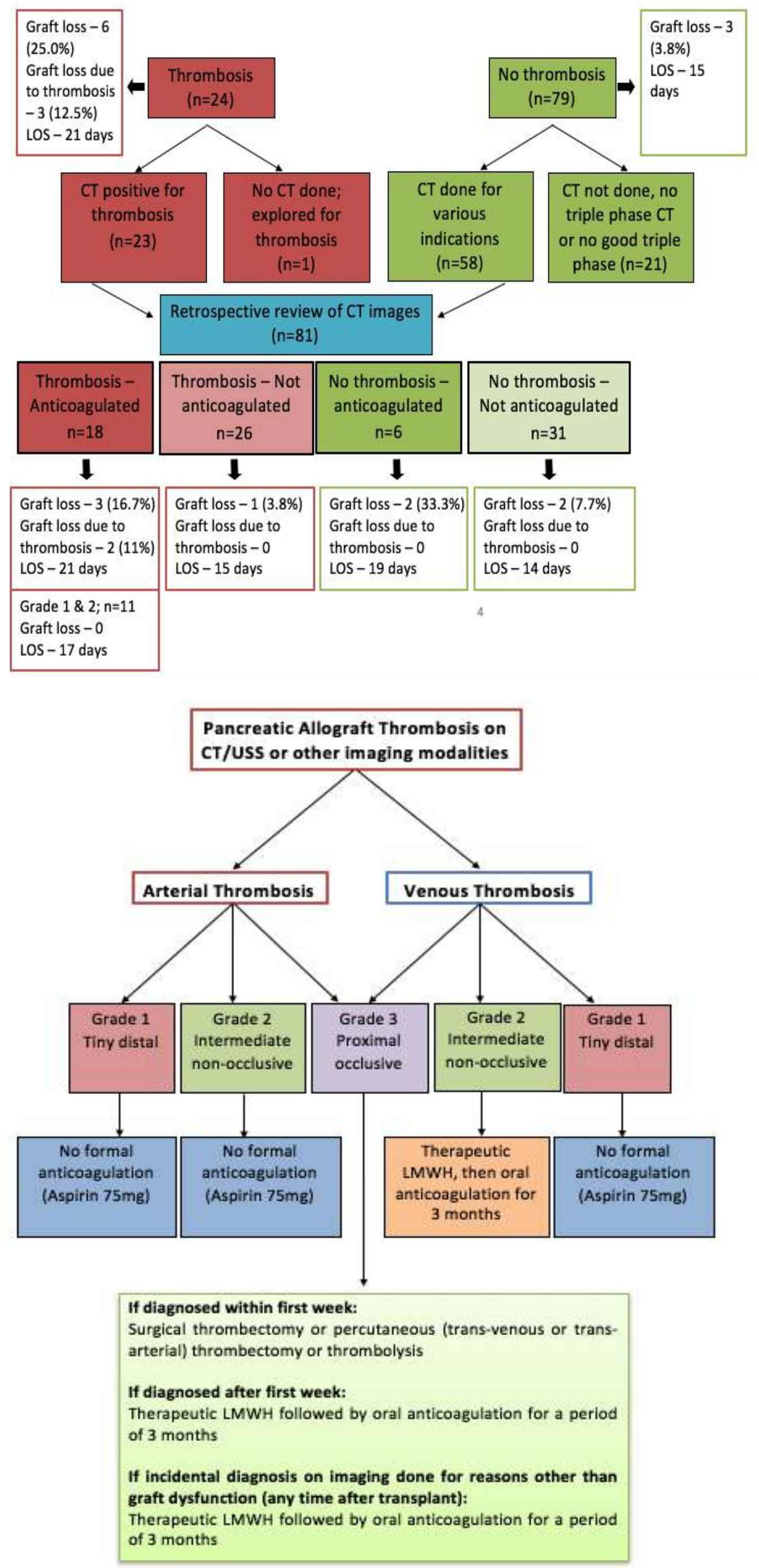